COMMUNICATIONS IN

NUMBER THEORY AND PHYSICS

Volume 2, Number 2, 325-347, 2008

\title{
Zeta stars
}

\author{
YASUo OHNo AND WADIM Zudilin
}

We present two new families of identities for the multiple zeta (star) values: The first one generalizes the formula $\zeta^{\star}\left(\{2\}_{n}, 1\right)=$ $2 \zeta(2 n+1)$, where $\{2\}_{n}$ denotes the $n$-tuple $(2,2, \ldots, 2)$, while the second family is a weighted analogue of Euler's formula $\sum_{l=2}^{n-1} \zeta(l$, $n-l)=\zeta(n)(n \geq 3)$.

\section{Introduction}

Although the structure of algebraic relations of the multiple zeta values $(\mathrm{MZVs})$

$$
\begin{aligned}
\zeta(s) & =\zeta\left(s_{1}, \ldots, s_{n}\right) \\
& =\sum_{a_{1}>\cdots>a_{n} \geq 1} \frac{1}{a_{1}^{s_{1}} \cdots a_{n}^{s_{n}}}, \quad s_{1}, \ldots, s_{n} \in\{1,2, \ldots\}, s_{1} \geq 2,
\end{aligned}
$$

got a conjectural description (by means of shuffle and stuffle, or harmonic, relations) and there was a big progress on obtaining the (expected) upper bounds for dimensions of the $\mathbb{Q}$-spaces spanned by MZVs of fixed weight $|\boldsymbol{s}|=\operatorname{wt}(\boldsymbol{s})=s_{1}+\cdots+s_{n}$ (recent works of Deligne and Goncharov [3] and Terasoma [16]), new parametric families of elegant identities for MZVs continue to come.

It is worth mentioning the deep relations between MZVs and quantum physics, knot theory, Galois representations of the fundamental group of $\mathbb{P}^{1} \backslash\{0,1, \infty\}$, connection formulae of differential equations and so on. For example, calculating counter-terms of Feynman diagrams usually gives us the MZVs, and this is a natural link between quantum field theory, number theory and knot theory (see $[2,12]$ ). The iterated integral representation of MZVs originates from the Knizhnik-Zamolodchikov (KZ) equation which appears in conformal (two-dimensional quantum) field theory. The formalized $\mathrm{KZ}$ equation is solved in terms of the iterated integral, and the ratio of two of its solutions turns out to be a generating series of MZVs which is called the Drinfeld associator $[4,11]$. 
Many of MZV identities have a simple form for an alternative model of zeta values, so-called multiple zeta star values (MZSVs or zeta stars), ${ }^{1}$

$$
\begin{aligned}
\zeta^{\star}(s) & =\zeta^{\star}\left(s_{1}, \ldots, s_{n}\right) \\
& =\sum_{a_{1} \geq \cdots \geq a_{n} \geq 1} \frac{1}{a_{1}^{s_{1}} \ldots a_{n}^{s_{n}}}, \quad s_{1}, \ldots, s_{n} \in\{1,2, \ldots\}, s_{1} \geq 2,
\end{aligned}
$$

see the discussion in (K. Ihara, J. Kajikawa, Y. Ohno and J. Okuda, MZSVs vs. $M Z V s$, in preparation). The equivalence of the models of MZVs (1) and MZSVs (2), in the sense that the $\mathbb{Q}$-spaces in $\mathbb{R}$ spanned by MZVs and by MZSVs of fixed weight coincide, is a well-known fact (it is an immediate consequence of Proposition 1 below). In our further discussion, we require some standard characteristics of the index $s$ in (1) and (2): the already defined weight $|s|$ and the depth (or the length) $\ell(s)=\operatorname{dep}(s)=n$. By an admissible index $s=\left(s_{1}, \ldots, s_{n}\right) \in \mathbb{Z}^{n}$, we mean an index with positive entries and $s_{1} \geq 2$ (the latter condition is necessary for the convergence of the series in (1) and (2)). We also assign these characteristics to an MZV $\zeta(s)$ or an MZSV $\zeta^{\star}(s)$ themselves, just speaking about the weight and depth of the multiple zeta (star) value, and calling it admissible if $s$ is an admissible index.

Apart from a simplicity of several identities in terms of MZSVs (a good example is the cyclic sum formula - cf. [15] versus [9]), these zeta values appear very naturally in the diophantine problems for the values of the classical Riemann zeta function $\zeta(s)$, especially due to the identities

$$
\begin{aligned}
\zeta^{\star}\left(\{2\}_{n}, 1\right) & =2 \zeta(2 n+1), \\
\zeta^{\star}\left(\{2\}_{n}\right) & =2\left(1-2^{1-2 n}\right) \zeta(2 n),
\end{aligned}
$$

where $\{2\}_{n}$ denotes the $n$-tuple $(2,2, \ldots, 2)$; the interested reader is referred to the papers by Zlobin [20-22] (we discuss these aspects at the end of Section 1). Identity (3a) also comes as a special case of the cyclic sum formula [15] while (3b) follows from a generating series argument $[1,22]$.

The starting goal of our project was to find a general form of identities (3a) and (3b), and on its way, we succeeded at least in generalizing (3a) (see the "two-one formula" accompanied with Theorems 1 and 2 below). Another result (Theorem 3) appearing as an auxiliary identity in

\footnotetext{
${ }^{1}$ They also appear as non-strict MZVs in [14], the name which is owed to the anonymous referee of that paper. Note also that the notation $\widetilde{\zeta}$ instead of $\zeta^{\star}$ was used in the 'Russian' literature [17, 22, 23].
} 
our deduction of Theorem 2, which is new and interesting by itself, is a "weighted" version of Euler's formula [5]

$$
\sum_{l=2}^{n-1} \zeta(l, n-l)=\zeta(n), \quad n \geq 3
$$

(the sum formula of depth 2 in the modern terminology).

In Section 1, we provide formulae for expressing the values (2) in terms of (1) and vice versa, and state our main results (Theorems 1 to 3 ). Section 2 is devoted to the proof of Theorem 1, while Theorems 2 and 3 are proved in Section 3 .

\section{Background and formulae for multiple zeta (star) values}

Let us first consider a simple recipe of passing from MZSVs to MZVs and vice versa.

Proposition 1. For any admissible index $\boldsymbol{s}=\left(s_{1}, s_{2}, \ldots, s_{n}\right)$, we have the (dual) relations

$$
\zeta^{\star}(\boldsymbol{s})=\sum_{\boldsymbol{p}} \zeta(\boldsymbol{p}) \quad \text { and } \quad \zeta(\boldsymbol{s})=\sum_{\boldsymbol{p}}(-1)^{\sigma(\boldsymbol{p})} \zeta^{\star}(\boldsymbol{p})
$$

where $\boldsymbol{p}$ runs through all indices of the form $\left(s_{1} \circ s_{2} \circ \cdots \circ s_{n}\right)$ with "०" being either the symbol "," or the sign "+", and the exponent $\sigma(\boldsymbol{p})$ denotes the number of signs "+" in $\boldsymbol{p}$. (The total number of such indices $\boldsymbol{p}$ is $2^{n-1}$.)

This statement is almost obvious (at least for the first identity in (5); the second one follows by the Möbius inversion). It also comes as a special case of Propositions 2 and 3 in [17] for generalized polylogarithms

(6) $\operatorname{Li}_{\boldsymbol{s}}(z)=\sum_{a_{1}>\cdots>a_{n} \geq 1} \frac{z^{a_{1}}}{a_{1}^{s_{1}} \cdots a_{n}^{s_{n}}}$ and $\operatorname{Le}_{\boldsymbol{s}}(z)=\sum_{a_{1} \geq \cdots \geq a_{n} \geq 1} \frac{z^{a_{1}}}{a_{1}^{s_{1}} \cdots a_{n}^{s_{n}}}$

at the point $z=1$.

The following identity was discovered experimentally when we searched for a generalization of (3a) (which is the particular case $l=1$ of our finding). It is a weighted analogue of the expressions on the right-hand sides in (5).

Two-one formula. For $k=0,1,2, \ldots$, denote $\mu_{2 k+1}=\left(\{2\}_{k}, 1\right)$. Then for any admissible index $s=\left(s_{1}, s_{2}, \ldots, s_{l}\right)$ with odd entries $s_{1}, \ldots, s_{l}$, the 
following identities are valid:

$$
\begin{aligned}
\zeta^{\star}\left(\mu_{s_{1}}, \mu_{s_{2}}, \ldots, \mu_{s_{l}}\right) & =\sum_{\boldsymbol{p}}(-1)^{\sigma(\boldsymbol{p})} 2^{l-\sigma(\boldsymbol{p})} \zeta^{\star}(\boldsymbol{p}) \\
& =\sum_{\boldsymbol{p}} 2^{l-\sigma(\boldsymbol{p})} \zeta(\boldsymbol{p}),
\end{aligned}
$$

where, as before, $\boldsymbol{p}$ runs through all indices of the form $\left(s_{1} \circ s_{2} \circ \cdots \circ s_{l}\right)$ with "о" being either the symbol "," or the sign "+", and the exponent $\sigma(\boldsymbol{p})$ denotes the number of signs "+" in $\boldsymbol{p}$.

Proof of the equality of the right-hand sides in (7a) and (7b). By Proposition 1 for the right-hand side in (7a), we have

$$
\begin{aligned}
& \sum_{\{\square=, \text { or }+\}}(-1)^{\#\{\square=+\}} 2^{l-\#\{\square=+\}} \zeta^{\star}\left(s_{1} \square s_{2} \square \cdots \square s_{l}\right) \\
& =\sum_{\{\square=, \text { or }+\}\{0=+ \text { or } \square\}}(-1)^{l-\#\{\circ=\square\}-1} 2^{\#\{\circ=\square\}+1} \zeta\left(s_{1} \circ s_{2} \circ \cdots \circ s_{l}\right),
\end{aligned}
$$

which in the notation $r=\#\{0=\square\}+1$ turns out to be

$$
\begin{aligned}
= & \sum_{n=1}^{l}\left(\sum_{r=n}^{l}\left(\begin{array}{c}
l-n \\
l-r
\end{array}\right)(-1)^{l-r} 2^{r}\right) \sum_{l-\#\{\circ=+\}=n} \zeta\left(s_{1} \circ s_{2} \circ \cdots \circ s_{l}\right) \\
= & \sum_{n=1}^{l}\left(\sum_{m=0}^{l-n}\left(\begin{array}{c}
l-n \\
l-n-m
\end{array}\right)(-1)^{l-n-m} 2^{n+m}\right) \\
& \times \sum_{n=1}^{\#\{=+\}=l-n} \zeta\left(s_{1} \circ s_{2} \circ \cdots \circ s_{l}\right) \\
= & \sum_{n=1}^{l} 2^{n} \sum_{\{0=+ \text { or }+\}} \zeta\left(s_{1} \circ s_{2} \circ \cdots \circ s_{l}\right) \\
= & \sum^{l-\#\{\circ=+\}} \zeta\left(s_{1} \circ s_{2} \circ \cdots \circ s_{l}\right),
\end{aligned}
$$

which is exactly the right-hand side of $(7 \mathrm{~b})$.

In spite of a nicely simple (but somehow unusual) form of the two-one formula, we cannot yet prove it in the full generality. The following two particular cases $\left(l=2\right.$, and $s_{1}=3, s_{2}=\cdots=s_{n-2}=1$ with $n=l+2 \geq$ 3 arbitrary) as well as our experimental results (for cases not included in the theorems below) strongly support the validity of identities (7a), (7b). 
Theorem 1. For any $n \geq 1$ and $1 \leq i \leq n$,

$$
\zeta^{\star}(\underbrace{2, \ldots, 2}_{i}, 1, \underbrace{2, \ldots, 2}_{n-i}, 1)=4 \zeta^{\star}(2 i+1,2 n-2 i+1)-2 \zeta(2 n+2) .
$$

Theorem 2. For any $n \geq 3$,

$$
\begin{aligned}
\zeta^{\star}(2, \underbrace{1, \ldots, 1}_{n-2}) & =\sum_{\{\circ=, \text { or }+\}} 2^{n-2-\#\{\circ=+\}} \zeta(3 \circ \underbrace{1 \circ \cdots \circ 1}_{n-3}) \\
& =\sum_{i=2}^{n-1} 2^{n-i} \sum_{e_{1}+e_{2}+\cdots+e_{n-i}=i-2} \zeta\left(3+e_{1}, 1+e_{2}, 1+e_{3}, \ldots, 1+e_{n-i}\right),
\end{aligned}
$$

where all $e_{j}$ are non-negative integers.

We deduce Theorem 2 from the following weighted analogue of Euler's formula (4).

Theorem 3 (Weighted sum formula). For any $n \geq 3$,

$$
\sum_{l=2}^{n-1} 2^{l} \zeta(l, n-l)=(n+1) \zeta(n)
$$

Before proceeding with our proofs of Theorems 1 to 3 , let us make some comments on the two-one formula.

The formula

$$
\zeta^{\star}\left(\left\{2,\{1\}_{m-1}\right\}_{n}, 1\right)=(m+1) \zeta((m+1) n+1)
$$

for any positive integers $m, n$ is known (two different proofs are given in $[15$, $22]$ ). If $m=1$ it is nothing but formula (3a), while if $m \geq 2$ then its lefthand side equals $\zeta^{\star}\left(\left\{\mu_{3},\left\{\mu_{1}\right\}_{m-2}\right\}_{n}, \mu_{1}\right)$. This together with the two-one formula mean that the corresponding right-hand side in (7a) (equivalently, in $(7 b))$ is expected to have a closed-form evaluation by means of the single zeta value $(m+1) \zeta((m+1) n+1)$, where the integers $m \geq 2$ and $n \geq 1$ are arbitrary. 
Using the integral representation of MZSVs,

$$
\zeta^{\star}(s)=\int \cdots \int_{[0,1]^{s_{1}+\cdots+s_{l}}} \frac{d x_{1} \cdots d x_{s_{1}+\cdots+s_{l}}}{\prod_{i=1}^{l}\left(1-x_{1} \cdots x_{s_{1}+\cdots+s_{i}}\right)}
$$

valid for any admissible index $s=\left(s_{1}, \ldots, s_{l}\right)$, we can write the right-hand side of (7a) as follows:

$$
2 \int \cdots \int_{[0,1]^{s_{1}+\cdots+s_{l}}} \frac{\prod_{i=1}^{l-1}\left(1+x_{1} \cdots x_{s_{1}+\cdots+s_{i}}\right)}{\prod_{i=1}^{l}\left(1-x_{1} \cdots x_{s_{1}+\cdots+s_{i}}\right)} d x_{1} \cdots d x_{s_{1}+\cdots+s_{l}} .
$$

The change of variable $y_{j}=x_{1} \cdots x_{j}$ for $j=1, \ldots, s_{1}+\cdots+s_{l}$ gives the integral

$$
\begin{aligned}
& \quad 2 \int \cdots \int \prod_{1>y_{1}>\cdots>y_{s_{1}+\cdots+s_{l}>0}}^{l-1}\left(\prod_{j=s_{1}+\cdots+s_{i-1}+1}^{s_{1}+\cdots+s_{i}-1} \frac{d y_{j}}{y_{j}} \cdot \frac{\left(1+y_{s_{1}+\cdots+s_{i}}\right) d y_{s_{1}+\cdots+s_{i}}}{\left(1-y_{s_{1}+\cdots+s_{i}}\right) y_{s_{1}+\cdots+s_{i}}}\right) \\
& \quad \times \prod_{j=s_{1}+\cdots+s_{l-1}+1}^{s_{1}+\cdots+s_{l}-1} \frac{d y_{j}}{y_{j}} \cdot \frac{d y_{s_{1}+\cdots+s_{l}}}{1-y_{s_{1}+\cdots+s_{l}}},
\end{aligned}
$$

where the empty sum $s_{1}+\cdots+s_{i-1}$ for $i=1$ is interpreted as 0 . Therefore, any of the two integrals in (11a), (11b) may replace the right-hand sides of $(7 \mathrm{a})$ or $(7 \mathrm{~b})$.

The case $l=1$ of the two-one formula is identity (3a). The case $l=2$ (Theorem 1) reads as

$$
\zeta^{\star}\left(\{2\}_{s_{1}}, 1,\{2\}_{s_{2}}, 1\right)=2 \zeta\left(2 s_{1}+2 s_{2}+2\right)+4 \zeta\left(2 s_{1}+1,2 s_{2}+1\right) .
$$

In particular, the latter identity implies

$$
\begin{aligned}
& \zeta^{\star}\left(\{2\}_{s_{1}}, 1,\{2\}_{s_{2}}, 1\right)+\zeta^{\star}\left(\{2\}_{s_{2}}, 1,\{2\}_{s_{1}}, 1\right) \\
& \quad=4 \zeta\left(2 s_{1}+2 s_{2}+2\right)+4 \zeta\left(2 s_{1}+1,2 s_{2}+1\right)+4 \zeta\left(2 s_{2}+1,2 s_{1}+1\right) \\
& \quad=4 \zeta\left(2 s_{1}+1\right) \zeta\left(2 s_{2}+1\right)=\zeta^{\star}\left(\{2\}_{s_{1}}, 1\right) \zeta^{\star}\left(\{2\}_{s_{2}}, 1\right)
\end{aligned}
$$

whenever $s_{1} \geq 1$ and $s_{2} \geq 1$.

On the right-hand side of (7a) and (7b), we have MZSVs and MZVs of length at most $l$, while the left-hand side involves a single zeta star attached to an index with entries 2 and 1 only (and the number of 1 's is equal to $l$ ); the latter circumstance is the reason of dubbing the formula as the two-one formula. 
We stress that neither the two-one formula nor its special cases treated in Theorems 1 and 2 is specializations of identities for polylogarithms (6) as may be seen from the fact $\operatorname{Le}_{2,1}(z) \neq 2 \operatorname{Le}_{3}(z)$ for $z \neq 1$ (cf. formula (3a) with $n=1$ ).

Zlobin's theorem [21, Theorem 3] implies that under certain natural restrictions on positive integer parameters $b_{i}>a_{i}, i=1, \ldots, m$, and $c_{j}, j=$ $1, \ldots, l$, and with a choice of integers $2 \leq r_{1}<r_{2}<\cdots<r_{l}=m$ such that $r_{j+1}-r_{j}$ is 1 or 2 for all $j=1, \ldots, l-1$, the multiple integral

$$
\int \cdots \int_{[0,1]^{m}} \frac{\prod_{j=1}^{m} x_{i}^{a_{i}-1}\left(1-x_{i}\right)^{b_{i}-a_{i}-1}}{\prod_{j=1}^{l}\left(1-z x_{1} x_{2} \cdots x_{r_{j}}\right)^{c_{j}}} d x_{1} \cdots d x_{m}
$$

is a $\mathbb{Q}\left[z^{-1}\right]$-linear combination of generalized polylogarithms $\operatorname{Le}_{\boldsymbol{s}}(z)$ with indices $s$ of weight at most $m$ and length at most $l$ whose entries are only 2 and 1. In several cases, the method in [21] allows to control the number of 1's in these indices (for instance, the case when only the zeta stars from (3a) occur after specialization $z=1$ is treated in [21] in details). The integrals (12) and formulae (7a) and (7b) may therefore become useful in diophantine study of multiple zeta (star) values of depth higher than 1.

\section{Depth 2 case of the two-one problem: Proof of Theorem 1}

For $a \geq c>0$, we define the harmonic sum

$$
H(a, c)=\sum_{\substack{j=1 \\ j \neq a}}^{c} \frac{1}{a-j}
$$

and interpret $H(\infty, c)$ and $H(a, 0)$ as zero.

Lemma 1. If $B \geq C$, we have

$$
\begin{aligned}
\sum_{\substack{A \geq a \geq B \\
C \geq c \geq D}} \frac{1}{a^{2} c}= & \sum_{C \geq c \geq D} \frac{H(B, c)-H(A+1, c)}{c^{2}} \\
& -\sum_{A \geq a \geq B} \frac{H(a, C)-H(a, D-1)}{a^{2}}+\delta_{B, C} \frac{2}{B^{3}} .
\end{aligned}
$$


Proof. It follows that

$$
\sum_{\substack{C \geq c \geq D \\ c \neq a}} \frac{1}{a-c}=H(a, C)-H(a, D-1)
$$

whenever $a \geq C$, and

$$
\sum_{\substack{A \geq a \geq B \\ a \neq c}}\left(\frac{1}{a-c}-\frac{1}{a}\right)=H(B, c)-H(A+1, c)+\delta_{c, B} \frac{1}{c}
$$

whenever $c \leq B$. Furthermore, for $a \neq c$, the following partial fraction decomposition is valid:

$$
\frac{1}{a^{2} c}=\left(\frac{1}{a-c}-\frac{1}{a}\right) \cdot \frac{1}{c^{2}}-\frac{1}{a-c} \cdot \frac{1}{a^{2}}
$$

Thus, under the condition $B \geq C$, we get

$$
\begin{aligned}
\sum_{\substack{A \geq a \geq B \\
C \geq c \geq D}} \frac{1}{a^{2} c}= & \sum_{\substack{A \geq a \geq B \\
C \geq c \geq D \\
a \neq c}}\left(\left(\frac{1}{a-c}-\frac{1}{a}\right) \cdot \frac{1}{c^{2}}-\frac{1}{a-c} \cdot \frac{1}{a^{2}}\right)+\delta_{B, C} \frac{1}{B^{3}} \\
= & \sum_{C \geq c \geq D} \frac{H(B, c)-H(A+1, c)}{c^{2}} \\
& -\sum_{A \geq a \geq B} \frac{H(a, C)-H(a, D-1)}{a^{2}}+\delta_{B, C} \frac{2}{B^{3}},
\end{aligned}
$$

which is the desired statement.

Remark 1. The proof of the cyclic sum theorem in [15] exploits the general forms of identities (15) and (14) which are

$$
\sum_{l=1}^{m-1} \frac{1}{a^{m+1-l} c^{l}}=\left(\frac{1}{a-c}-\frac{1}{a}\right) \cdot \frac{1}{c^{m}}-\frac{1}{a-c} \cdot \frac{1}{a^{m}}
$$


and

$$
\begin{aligned}
\sum_{\substack{A \geq a \geq B \\
C \geq c \geq D}} \sum_{l=1}^{m-1} \frac{1}{a^{m+1-l} c^{l}}= & \sum_{C \geq c \geq D} \frac{H(B, c)-H(A+1, c)}{c^{m}} \\
& -\sum_{A \geq a \geq B} \frac{H(a, C)-H(a, D-1)}{a^{m}}+\delta_{B, C} \frac{m}{B^{m+1}},
\end{aligned}
$$

respectively, although the function (13) is not used there in an explicit form (it was introduced later by D. Zagier in his unpublished note on the proof in [15]). We are wondering if the two-one formula may be generalized to some kind of "multiple cyclic formula."

Lemma 2. For any $i \geq 1$ and $j \geq 0$ we have

$$
\zeta^{\star}(2 i+1, \underbrace{2, \ldots, 2}_{j}, 1)=-\sum_{a_{0} \geq a_{1} \geq \cdots \geq a_{j} \geq 1} \frac{H\left(a_{0}+1, a_{j}\right)}{a_{0}^{2 i+1} a_{1}^{2} \cdots a_{j}^{2}}+2 \zeta^{\star}(2 i+1,2 j+1) .
$$

Proof. If $j \geq 1$, we apply Lemma 1 with $a=a_{1}$ and $c=a_{j+1}$

$$
\begin{gathered}
\zeta^{\star}(2 i+1, \underbrace{2, \ldots, 2}_{j}, 1)=\sum_{a_{0} \geq a_{1} \geq \cdots \geq a_{j+1} \geq 1} \frac{1}{a_{0}^{2 i+1} a_{1}^{2} \cdots a_{j}^{2} a_{j+1}} \\
=\sum_{a_{0} \geq a_{2} \geq \cdots \geq a_{j+1} \geq 1} \frac{H\left(a_{2}, a_{j+1}\right)-H\left(a_{0}+1, a_{j+1}\right)}{a_{0}^{2 i+1} a_{2}^{2} \cdots a_{j}^{2} a_{j+1}^{2}} \\
-\sum_{a_{0} \geq a_{1} \geq \cdots \geq a_{j} \geq 1} \frac{H\left(a_{1}, a_{j}\right)}{a_{0}^{2 i+1} a_{1}^{2} a_{2}^{2} \cdots a_{j}^{2}}+2 \sum_{a_{0} \geq a_{1} \geq 1} \frac{1}{a_{0}^{2 i+1} a_{1}^{2 j+1}} \\
=-\sum_{a_{0} \geq a_{1} \geq \cdots \geq a_{j} \geq 1} \frac{H\left(a_{0}+1, a_{j}\right)}{a_{0}^{2 i+1} a_{1}^{2} \cdots a_{j}^{2}}+2 \zeta^{\star}(2 i+1,2 j+1) .
\end{gathered}
$$

Although the application of (14) is only possible if $j \geq 1$, it is easy to see that the resulting formula (16) trivially holds for $j=0$, since

$$
\sum_{a_{0} \geq 1} \frac{H\left(a_{0}+1, a_{0}\right)}{a_{0}^{2 i+1}}=\sum_{a_{0}>b \geq 0} \frac{1}{a_{0}^{2 i+1}\left(a_{0}-b\right)}=\sum_{a_{0} \geq a_{1} \geq 1} \frac{1}{a_{0}^{2 i+1} a_{1}}=\zeta^{\star}(2 i+1,1)
$$

in this case.

We will also need the following formula whose proof is just changing the condition $a_{0} \geq a_{1}$ by $a_{0}>a_{1}$ in (17). 
Lemma 3. For any $i \geq 1$ and $j \geq 0$ we have

$$
\sum_{a_{0}>a_{1} \geq \cdots \geq a_{j+1} \geq 1}\left(\frac{1}{a_{0}-a_{j+1}}-\frac{1}{a_{0}}\right) \frac{1}{a_{0}^{2 i-1} a_{1}^{2} \cdots a_{j+1}^{2}}=2 \zeta(2 i+1,2 j+1) .
$$

Proof. We proceed as in (17)

$$
\begin{aligned}
& \sum_{a_{0}>a_{1} \geq \cdots \geq a_{j+1} \geq 1} \frac{1}{a_{0}^{2 i+1} a_{1}^{2} \cdots a_{j}^{2} a_{j+1}} \\
=- & \sum_{a_{0}>a_{1} \geq \cdots \geq a_{j} \geq 1} \frac{H\left(a_{0}, a_{j}\right)}{a_{0}^{2 i+1} a_{1}^{2} \cdots a_{j}^{2}}+2 \sum_{a_{0}>a_{1} \geq 1} \frac{1}{a_{0}^{2 i+1} a_{1}^{2 j+1}} \\
=- & \sum_{a_{0}>a_{1} \geq \cdots \geq a_{j+1} \geq 1} \frac{1}{a_{0}^{2 i+1} a_{1}^{2} \cdots a_{j}^{2}\left(a_{0}-a_{j+1}\right)}+2 \zeta(2 i+1,2 j+1) ;
\end{aligned}
$$

again the formula remains valid for $j=0$. Then, we use the identity

$$
\begin{aligned}
\frac{1}{a_{0}^{2 i+1} a_{j+1}}+\frac{1}{a_{0}^{2 i+1}\left(a_{0}-a_{j+1}\right)} & =\frac{1}{a_{0}^{2 i} a_{j+1}\left(a_{0}-a_{j+1}\right)} \\
& =\left(\frac{1}{a_{0}-a_{j+1}}-\frac{1}{a_{0}}\right) \frac{1}{a_{0}^{2 i-1} a_{j+1}^{2}}
\end{aligned}
$$

to conclude with the desired claim.

Lemma 4. Given $n \geq 1$, for any $1 \leq i \leq n$, the following identity holds:

$$
\begin{aligned}
& \zeta^{\star}(\underbrace{2, \ldots, 2}_{i}, 1, \underbrace{2, \ldots, 2}_{n-i}, 1)=4 \zeta^{\star}(2 i+1,2 n-2 i+1) \\
& \quad+\sum_{a_{0} \geq \cdots \geq a_{n} \geq 1} \frac{H\left(a_{0}, a_{i}-1\right)}{a_{0}^{2} \cdots a_{n-1}^{2} a_{n}}-2 \sum_{a_{0} \geq a_{i+1} \geq \cdots \geq a_{n} \geq 1} \frac{H\left(a_{0}+1, a_{n}\right)}{a_{0}^{2 i+1} a_{i+1}^{2} \cdots a_{n}^{2}}
\end{aligned}
$$

Proof. We use Lemma 1 with $a=a_{0}$ and $c=a_{i}$

$$
\begin{aligned}
\zeta^{\star} & (\underbrace{2, \ldots, 2}_{i}, 1, \underbrace{2, \ldots, 2}_{n-i}, 1) \\
& =\sum_{a_{0} \geq \cdots \geq a_{i} \geq a_{i+1} \geq \cdots \geq a_{n+1} \geq 1} \frac{1}{a_{1}^{2} \cdots a_{i-1}^{2} a_{i} a_{i+1}^{2} \cdots a_{n}^{2} a_{n+1}}
\end{aligned}
$$




$$
\begin{aligned}
& =\sum_{a_{1} \geq \cdots \geq a_{i-1} \geq a_{i} \geq a_{i+1} \geq \cdots \geq a_{n+1} \geq 1} \frac{H\left(a_{1}, a_{i}\right)}{a_{1}^{2} \cdots a_{i-1}^{2} a_{i}^{2} a_{i+1}^{2} \cdots a_{n}^{2} a_{n+1}} \\
& -\sum_{a_{0} \geq a_{1} \geq \cdots \geq a_{i-1} \geq a_{i+1} \geq \cdots \geq a_{n+1} \geq 1} \frac{H\left(a_{0}, a_{i-1}\right)-H\left(a_{0}, a_{i+1}-1\right)}{a_{0}^{2} a_{1}^{2} \cdots a_{i-1}^{2} a_{i+1}^{2} \cdots a_{n}^{2} a_{n+1}} \\
& +2 \sum_{a_{0} \geq a_{i+1} \geq \cdots \geq a_{n+1} \geq 1} \frac{1}{a_{0}^{2 i+1} a_{i+1}^{2} \cdots a_{n}^{2} a_{n+1}} \\
& =\sum_{a_{0} \geq a_{1} \geq \cdots \geq a_{i-1} \geq a_{i+1} \geq \cdots \geq a_{n+1} \geq 1} \frac{H\left(a_{0}, a_{i+1}-1\right)}{a_{0}^{2} a_{1}^{2} \cdots a_{i-1}^{2} a_{i+1}^{2} \cdots a_{n}^{2} a_{n+1}} \\
& +2 \zeta^{\star}(2 i+1, \underbrace{2, \ldots, 2}_{n-i}, 1) \\
& \sum_{a_{0} \geq \cdots \geq a_{n} \geq 1} \frac{H\left(a_{0}, a_{i}-1\right)}{a_{0}^{2} \cdots a_{n-1}^{2} a_{n}}+2 \zeta^{\star}(2 i+1, \underbrace{2, \ldots, 2}_{n-i}, 1),
\end{aligned}
$$

and for the latter zeta star term, we apply Lemma 2.

Lemma 5. We have

$$
\zeta^{\star}(\underbrace{2, \ldots, 2}_{n}, 1,1)=4 \zeta^{\star}(2 n+1,1)-2 \zeta(2 n+2) .
$$

To prove Lemma 5, we will require an additional identity.

Lemma 6. For any positive integers $l$ and $m$ satisfying $l>m$, the following identity is valid:

$$
\sum_{b=1}^{m} \frac{H(l, b)}{l-b}=\sum_{a=l}^{\infty}\left(\frac{1}{a-m}-\frac{1}{a}\right) H(a+1, m+1) .
$$

Proof. For the right-hand side in (19), we have

$$
\begin{gathered}
\sum_{a=l}^{\infty}\left(\frac{1}{a-m}-\frac{1}{a}\right) H(a+1, m+1) \\
\quad=\sum_{a=l}^{\infty} \sum_{d=0}^{m}\left(\frac{1}{a-m}-\frac{1}{a}\right) \frac{1}{a-d}
\end{gathered}
$$




$$
\begin{aligned}
& =\sum_{a=l}^{\infty} \sum_{d=0}^{m}\left(\frac{1}{(a-m)(a-d)}-\frac{1}{a(a-d)}\right) \\
& =\sum_{a=l}^{\infty}\left(\frac{1}{(a-m)^{2}}+\sum_{d=0}^{m-1} \frac{1}{m-d}\left(\frac{1}{a-m}-\frac{1}{a-d}\right)\right. \\
& \left.-\frac{1}{a^{2}}-\sum_{d=1}^{m} \frac{1}{d}\left(\frac{1}{a-d}-\frac{1}{a}\right)\right) \\
& =\sum_{b=1}^{m} \frac{1}{(l-b)^{2}}+\sum_{d=0}^{m-1} \frac{1}{m-d} \sum_{a=l}^{\infty}\left(\frac{1}{a-m}-\frac{1}{a-d}\right) \\
& -\sum_{d=1}^{m} \frac{1}{d} \sum_{a=l}^{\infty}\left(\frac{1}{a-d}-\frac{1}{a}\right) \\
& =\sum_{b=1}^{m} \frac{1}{(l-b)^{2}}+\sum_{d=0}^{m-1} \frac{1}{m-d} \sum_{c=d+1}^{m} \frac{1}{l-c}-\sum_{d=1}^{m} \frac{1}{d} \sum_{c=1}^{d} \frac{1}{l-c} \\
& =\sum_{b=1}^{m} \frac{1}{(l-b)^{2}}+\sum_{b=1}^{m} \frac{1}{b} \sum_{c=m-b+1}^{m} \frac{1}{l-c}-\sum_{b=1}^{m} \frac{1}{b} \sum_{c=1}^{b} \frac{1}{l-c} \\
& =\sum_{b=1}^{m} \frac{1}{(l-b)^{2}}+\sum_{b=1}^{m} \frac{1}{b}\left(\sum_{c=m-b+1}^{m}-\sum_{c=1}^{b}\right) \frac{1}{l-c},
\end{aligned}
$$

while the right-hand side can be written as follows:

$$
\begin{aligned}
\sum_{b=1}^{m} \frac{H(l, b)}{l-b} & =\sum_{b=1}^{m} \sum_{c=1}^{b} \frac{1}{(l-b)(l-c)} \\
& =\sum_{b=1}^{m} \frac{1}{(l-b)^{2}}+\sum_{b=1}^{m} \sum_{c=1}^{b-1} \frac{1}{b-c}\left(\frac{1}{l-b}-\frac{1}{l-c}\right) \\
& =\sum_{b=1}^{m} \frac{1}{(l-b)^{2}}+\sum_{d=1}^{m} \sum_{c=1}^{d-1} \frac{1}{d-c} \frac{1}{l-d}-\sum_{d=1}^{m} \sum_{c=1}^{d-1} \frac{1}{d-c} \frac{1}{l-c} \\
& =\sum_{b=1}^{m} \frac{1}{(l-b)^{2}}+\sum_{d=1}^{m} \frac{1}{l-d} \sum_{c=1}^{d-1} \frac{1}{d-c}-\sum_{c=1}^{m-1} \frac{1}{l-c} \sum_{d=c+1}^{m} \frac{1}{d-c}
\end{aligned}
$$




$$
\begin{aligned}
& =\sum_{b=1}^{m} \frac{1}{(l-b)^{2}}+\sum_{d=1}^{m} \frac{1}{l-d} \sum_{b=1}^{d-1} \frac{1}{b}-\sum_{c=1}^{m-1} \frac{1}{l-c} \sum_{b=1}^{m-c} \frac{1}{b} \\
& =\sum_{b=1}^{m} \frac{1}{(l-b)^{2}}+\sum_{b=1}^{m} \frac{1}{b} \sum_{d=b+1}^{m} \frac{1}{l-d}-\sum_{b=1}^{m} \frac{1}{b} \sum_{c=1}^{m-b} \frac{1}{l-c} \\
& =\sum_{b=1}^{m} \frac{1}{(l-b)^{2}}+\sum_{b=1}^{m} \frac{1}{b}\left(\sum_{c=b+1}^{m}-\sum_{c=1}^{m-b}\right) \frac{1}{l-c} .
\end{aligned}
$$

Comparing the right-hand sides of the resulting expressions in (20) and (21) and using

$$
\sum_{c=m-b+1}^{m}-\sum_{c=1}^{b}=\left(\sum_{c=1}^{m}-\sum_{c=1}^{m-b}\right)-\left(\sum_{c=1}^{m}-\sum_{c=b+1}^{m}\right)=\sum_{c=b+1}^{m}-\sum_{c=1}^{m-b}
$$

we derive the required identity (19).

Proof of Lemma 5. We are first interested in the finite multiple sum

$$
\begin{aligned}
F_{n}(a, b)= & \sum_{\substack{a_{0} \geq \cdots \geq a_{n} \\
a_{0} \leq a, a_{n}>b}} \frac{1}{a_{0}^{2} \cdots a_{n-1}^{2} a_{n}} \\
= & \sum_{\substack{a_{1} \geq \cdots \geq a_{n} \\
a_{1} \leq a, a_{n}>b}} \frac{H\left(a_{1}, a_{n}\right)-H\left(a+1, a_{n}\right)}{a_{1}^{2} \cdots a_{n-1}^{2} a_{n}^{2}} \\
& -\sum_{\substack{a_{0} \geq \cdots \geq a_{n-1} \\
a_{0} \leq a, a_{n-1}>b}} \frac{H\left(a_{0}, a_{n-1}\right)-H\left(a_{0}, b\right)}{a_{0}^{2} a_{1}^{2} \cdots a_{n-1}^{2}}+2 \sum_{a \geq a_{0}>b} \frac{1}{a_{0}^{2 n+1}} \\
= & \sum_{\substack{a_{0} \geq \cdots \geq a_{n-1} \\
a_{0} \leq a, a_{n-1}>b}} \frac{H\left(a_{0}, b\right)-H\left(a+1, a_{n-1}\right)}{a_{0}^{2} a_{1}^{2} \cdots a_{n-1}^{2}}+2 \sum_{a \geq a_{0}>b} \frac{1}{a_{0}^{2 n+1}} .
\end{aligned}
$$

Multiplying both expressions for the sum $F_{n}(a, b)$ in $(22)$ by $1 /(a-b)-$ $1 /(a-b+1)$, notifying that

$$
\sum_{a \geq a_{0}}\left(\frac{1}{a-b}-\frac{1}{a-b+1}\right)=\frac{1}{a_{0}-b}
$$


and

$$
\sum_{b=1}^{a_{n}-1}\left(\frac{1}{a-b}-\frac{1}{a-b+1}\right)=\frac{1}{a-a_{n}+1}-\frac{1}{a}
$$

and summing over all $a$ and $b, a>b \geq 1$, we obtain

$$
\begin{aligned}
& \sum_{a_{0} \geq \cdots \geq a_{n}>b \geq 1} \frac{1}{\left(a_{0}-b\right) a_{0}^{2} \cdots a_{n-1}^{2} a_{n}}=\sum_{a_{0} \geq \cdots \geq a_{n-1}>b \geq 1} \frac{H\left(a_{0}, b\right)}{\left(a_{0}-b\right) a_{0}^{2} a_{1}^{2} \cdots a_{n-1}^{2}} \\
& \quad-\sum_{a \geq a_{0} \geq \cdots \geq a_{n-1}>1}\left(\frac{1}{a-a_{n-1}+1}-\frac{1}{a}\right) \frac{H\left(a+1, a_{n-1}\right)}{a_{0}^{2} a_{1}^{2} \cdots a_{n-1}^{2}} \\
& \quad+2 \sum_{a_{0}>b \geq 1} \frac{1}{\left(a_{0}-b\right) a_{0}^{2 n+1}} .
\end{aligned}
$$

Applying Lemma 6 with $l=a_{0}$ and $m=a_{n-1}-1$, we derive from (23) that

$$
\sum_{a_{0} \geq \cdots \geq a_{n}>b \geq 1} \frac{1}{\left(a_{0}-b\right) a_{0}^{2} \cdots a_{n-1}^{2} a_{n}}=2 \sum_{a_{0}>b \geq 1} \frac{1}{\left(a_{0}-b\right) a_{0}^{2 n+1}} .
$$

Finally, from Lemma 4 in the case $i=n$, we obtain

$$
\begin{aligned}
\zeta^{\star}(\underbrace{2, \ldots, 2}_{n}, 1,1)= & 4 \zeta^{\star}(2 n+1,1)+2 \sum_{a_{0}>b \geq 1} \frac{1}{\left(a_{0}-b\right) a_{0}^{2 n+1}} \\
& -2 \sum_{a_{0}>b \geq 0} \frac{1}{\left(a_{0}-b\right) a_{0}^{2 n+1}}=4 \zeta^{\star}(2 n+1,1)-2 \zeta(2 n+2),
\end{aligned}
$$

which is the desired identity.

Lemma 7. For $1 \leq i<n$, we have

$$
\begin{aligned}
& \sum_{a_{0} \geq \cdots \geq a_{n} \geq 1} \frac{H\left(a_{0}, a_{i}-1\right)-H\left(a_{0}, a_{i+1}-1\right)}{a_{0}^{2} \cdots a_{n-1}^{2} a_{n}} \\
= & 2 \sum_{a_{0}>a_{i+1} \geq \cdots \geq a_{n} \geq 1} \frac{H\left(a_{0}, a_{n}\right)}{a_{0}^{2 i+1} a_{i+1}^{2} \cdots a_{n}^{2}} \\
& -\sum_{a_{0} \geq \cdots \geq a_{n} \geq 1}\left(\frac{H\left(a_{0}, a_{n}\right)}{a_{0}^{2} \cdots a_{i-1}^{2} a_{i} a_{i+1}^{2} \cdots a_{n}^{2}}-\frac{H\left(a_{0}, a_{n}\right)}{a_{0}^{2} \cdots a_{i}^{2} a_{i+1} a_{i+2}^{2} \cdots a_{n}^{2}}\right) .
\end{aligned}
$$


Proof. We use the identity in $(22)$ for $F_{i}\left(a, a_{i+1}-1\right)$ (that is, for $n$ replaced by $i$ and $b$ replaced by $\left.a_{i+1}-1\right)$. Multiplying it by $1 /\left(a_{i+1}^{2} \cdots a_{n}^{2}\right)$ and summing over $a_{i+1}, \ldots, a_{n+1}$ satisfying $a_{i+1} \geq \cdots \geq a_{n} \geq a_{n+1}$ result in the following identity:

$$
\begin{aligned}
\widetilde{F}_{i}\left(a, a_{n+1}\right)= & \sum_{\substack{a_{0} \geq \cdots \geq a_{i} \geq a_{i+1} \geq \cdots \geq a_{n} \\
a_{0} \leq a, a_{n} \geq a_{n+1}}} \frac{1}{a_{0}^{2} \cdots a_{i-1}^{2} a_{i} a_{i+1}^{2} \cdots a_{n}^{2}} \\
= & \sum_{\substack{a_{1} \geq \cdots \geq a_{i} \geq a_{i+1} \geq \cdots \geq a_{n} \\
a_{1} \leq a, a_{n} \geq a_{n+1}}} \frac{H\left(a_{1}, a_{i+1}-1\right)-H\left(a+1, a_{i}\right)}{a_{1}^{2} \cdots a_{n}^{2}} \\
& +2 \sum_{\substack{a_{1} \geq a_{i+1} \geq \cdots \geq a_{n} \\
a_{1} \leq a, a_{n} \geq a_{n+1}}} \frac{1}{a_{1}^{2 i+1} a_{i+1}^{2} \cdots a_{n}^{2}}
\end{aligned}
$$

As before, we multiply both sides by $1 /\left(a-a_{n+1}\right)-1 /\left(a-a_{n+1}+1\right)$ and sum over all $a \geq a_{n+1} \geq 1$ to obtain

$$
\begin{aligned}
& \sum_{a_{0} \geq \cdots \geq a_{n} \geq 1} \frac{H\left(a_{0}, a_{n}\right)}{a_{0}^{2} \cdots a_{i-1}^{2} a_{i} a_{i+1}^{2} \cdots a_{n}^{2}} \\
= & \sum_{\substack{a_{0} \geq \cdots \geq a_{n} \geq a_{n+1} \geq 1 \\
a_{0} \neq a_{n+1}}} \frac{1}{\left(a_{0}-a_{n+1}\right) a_{0}^{2} \cdots a_{i-1}^{2} a_{i} a_{i+1}^{2} \cdots a_{n}^{2}} \\
= & \sum_{\substack{a_{1} \geq \cdots \geq a_{n} \geq a_{n+1} \geq 1 \\
a_{1} \neq a_{n+1}}} \frac{H\left(a_{1}, a_{i+1}-1\right)}{\left(a_{1}-a_{n+1}\right) a_{1}^{2} \cdots a_{n-1}^{2} a_{n}^{2}} \\
& -\sum_{\substack{a \geq a_{1} \geq \cdots \geq a_{n} \geq 1 \\
a \neq a_{n}}}\left(\frac{1}{a-a_{n}}-\frac{1}{a}\right) \frac{H\left(a+1, a_{i}\right)}{a_{1}^{2} \cdots a_{n-1}^{2} a_{n}^{2}} \\
& +2 \sum_{\substack{a_{1} \geq a_{i+1} \geq \cdots \geq a_{n+1} \geq 1 \\
a_{1} \neq a_{n+1}}} \frac{1}{\left(a_{1}-a_{n+1}\right) a_{1}^{2 i+1} a_{i+1}^{2} \cdots a_{n}^{2}}
\end{aligned}
$$

(we set $a_{0}=a$ in the second sum)

$$
=\sum_{\substack{a_{1} \geq \cdots \geq a_{n} \geq a_{n+1} \geq 1 \\ a_{1} \neq a_{n+1}}} \frac{H\left(a_{1}, a_{i+1}-1\right)}{\left(a_{1}-a_{n+1}\right) a_{1}^{2} \cdots a_{n-1}^{2} a_{n}^{2}}
$$




$$
\begin{aligned}
& -\sum_{\substack{a_{0} \geq a_{1} \geq \cdots \geq a_{n} \geq 1 \\
a_{0} \neq a_{n}}}\left(\frac{1}{a_{0}-a_{n}}-\frac{1}{a_{0}}\right) \frac{H\left(a_{0}, a_{i}-1\right)+1 / a_{0}}{a_{1}^{2} \cdots a_{n-1}^{2} a_{n}^{2}} \\
& +2 \sum_{\substack{a_{1} \geq a_{i+1} \geq \cdots \geq a_{n} \geq 1 \\
a_{0} \geq \cdots \geq a_{n} \geq 1 \\
a_{0} \neq a_{n}}} \frac{H\left(a_{1}, a_{n}\right)}{a_{1}^{2 i+1} a_{i+1}^{2} \cdots a_{n}^{2}} \\
& -\sum_{\substack{a_{0} \geq a_{1} \geq \cdots \geq a_{0} \geq 1 \\
a_{0} \neq a_{n}}}\left(\frac{1}{\left.a_{0}-a_{n}\right) a_{0}^{2} \cdots a_{n-1}^{2}}-\frac{1}{a_{0}-a_{n}}\right) \frac{H\left(a_{0}, a_{i}-1\right)+1 / a_{0}}{a_{1}^{2} \cdots a_{n-1}^{2} a_{n}^{2}} \\
& +2 \sum_{\substack{a_{0} \geq a_{i+1} \geq \cdots \geq a_{n} \geq 1 \\
a_{0}^{2 i+1} a_{i+1}^{2} \cdots a_{n}^{2}}}
\end{aligned}
$$

Using the partial fraction decomposition (15) with $x=a_{0}$ and $y=a_{n}$ reduces the latter expression to

$$
\begin{aligned}
& \sum_{a_{0} \geq \cdots \geq a_{n} \geq 1} \frac{H\left(a_{0}, a_{n}\right)}{a_{0}^{2} \cdots a_{i-1}^{2} a_{i} a_{i+1}^{2} \cdots a_{n}^{2}}=-\sum_{\substack{a_{0} \geq \cdots \geq a_{n} \geq 1 \\
a_{0} \neq a_{n}}} \frac{H\left(a_{0}, a_{i}-1\right)}{a_{0}^{2} \cdots a_{n-1}^{2} a_{n}} \\
& \quad-\sum_{\substack{a_{0} \geq a_{1} \geq \cdots \geq a_{n} \geq 1 \\
a_{0} \neq a_{n}}}\left(\frac{1}{a_{0}-a_{n}}-\frac{1}{a_{0}}\right) \frac{1 / a_{0}}{a_{1}^{2} \cdots a_{n-1}^{2} a_{n}^{2}} \\
& \quad+2 \sum_{a_{0} \geq a_{i+1} \geq \cdots \geq a_{n} \geq 1} \frac{H\left(a_{0}, a_{n}\right)}{a_{0}^{2 i+1} a_{i+1}^{2} \cdots a_{n}^{2}} \\
& =-\sum_{a_{0} \geq \cdots \geq a_{n} \geq 1} \frac{H\left(a_{0}, a_{i}-1\right)}{a_{0}^{2} \cdots a_{n-1}^{2} a_{n}}+\sum_{a_{0} \geq 1} \frac{H\left(a_{0}, a_{0}-1\right)}{a_{0}^{2 n+1}} \\
& -\sum_{a_{0} \geq a_{1} \geq \cdots \geq a_{n} \geq 1} \frac{1}{\left(a_{0} \neq a_{n}-a_{n}\right) a_{0} a_{1}^{2} \cdots a_{n}^{2}}+\sum_{a_{0} \geq a_{1} \geq \cdots \geq a_{n} \geq 1} \frac{1}{a_{0}^{2} a_{1}^{2} \cdots a_{n}^{2}} \\
& -\sum_{a_{0} \geq 1} \frac{1}{a_{0}^{2 n+2}}+2 \sum_{a_{0} \geq a_{i+1} \geq \cdots \geq a_{n} \geq 1} \frac{H\left(a_{0}, a_{n}\right)}{a_{0}^{2 i+1} a_{i+1}^{2} \cdots a_{n}^{2}} .
\end{aligned}
$$


Subtracting now the expression in (25) from the one corresponding to the $i$ replaced by $i+1$, we finally obtain

$$
\begin{aligned}
& \sum_{a_{0} \geq \cdots \geq a_{n} \geq 1}\left(\frac{H\left(a_{0}, a_{n}\right)}{a_{0}^{2} \cdots a_{i}^{2} a_{i+1} a_{i+2}^{2} \cdots a_{n}^{2}}-\frac{H\left(a_{0}, a_{n}\right)}{a_{0}^{2} \cdots a_{i-1}^{2} a_{i} a_{i+1}^{2} \cdots a_{n}^{2}}\right) \\
& =\sum_{a_{0} \geq \cdots \geq a_{n} \geq 1} \frac{H\left(a_{0}, a_{i}-1\right)-H\left(a_{0}, a_{i+1}-1\right)}{a_{0}^{2} \cdots a_{n-1}^{2} a_{n}} \\
& \quad+2 \sum_{a_{0} \geq a_{i+2} \geq \cdots \geq a_{n} \geq 1} \frac{H\left(a_{0}, a_{n}\right)}{a_{0}^{2 i+3} a_{i+2}^{2} \cdots a_{n}^{2}} \\
& \quad-2 \sum_{a_{0} \geq a_{i+1} \geq \cdots \geq a_{n} \geq 1} \frac{H\left(a_{0}, a_{n}\right)}{a_{0}^{2 i+1} a_{i+1}^{2} \cdots a_{n}^{2}},
\end{aligned}
$$

which gives us the required formula (24).

Lemma 8. For $0 \leq i<n$, we have

$$
\begin{aligned}
& \sum_{a_{0} \geq \cdots \geq a_{n} \geq 1}\left(\frac{H\left(a_{0}, a_{n}\right)}{a_{0}^{2} \cdots a_{i-1}^{2} a_{i} a_{i+1}^{2} \cdots a_{n}^{2}}-\frac{H\left(a_{0}, a_{n}\right)}{a_{0}^{2} \cdots a_{i}^{2} a_{i+1} a_{i+2}^{2} \cdots a_{n}^{2}}\right) \\
=\zeta^{\star} & (\underbrace{2, \ldots, 2}_{i+1}, 1, \underbrace{2, \ldots, 2}_{n-i-1}, 1)-2 \zeta(2 n+2) .
\end{aligned}
$$

Proof. We apply Lemma 1 , this time with $a=a_{0}$ and $c=a_{n+1}$

$$
\begin{aligned}
\zeta^{\star}(\underbrace{2, \ldots, 2}_{i+1}, 1, \underbrace{2, \ldots, 2}_{n-i-1}, 1)=\sum_{a_{0} \geq \cdots \geq a_{n+1} \geq 1} \frac{1}{a_{0}^{2} \cdots a_{i}^{2} a_{i+1} a_{i+2}^{2} \cdots a_{n}^{2} a_{n+1}} \\
=\sum_{a_{1} \geq \cdots \geq a_{n+1} \geq 1} \frac{H\left(a_{1}, a_{n+1}\right)}{a_{1}^{2} \cdots a_{i}^{2} a_{i+1} a_{i+2}^{2} \cdots a_{n+1}^{2}} \\
\quad-\sum_{a_{0} \geq \cdots \geq a_{n} \geq 1} \frac{H\left(a_{0}, a_{n}\right)}{a_{0}^{2} \cdots a_{i}^{2} a_{i+1} a_{i+2}^{2} \cdots a_{n}^{2}}+2 \zeta(2 n+2) \\
=\sum_{a_{0} \geq \cdots \geq a_{n} \geq 1} \frac{H\left(a_{0}, a_{n}\right)}{a_{0}^{2} \cdots a_{i-1}^{2} a_{i} a_{i+1}^{2} \cdots a_{n}^{2}} \\
\quad-\sum_{a_{0} \geq \cdots \geq a_{n} \geq 1} \frac{H\left(a_{0}, a_{n}\right)}{a_{0}^{2} \cdots a_{i}^{2} a_{i+1} a_{i+2}^{2} \cdots a_{n}^{2}}+2 \zeta(2 n+2),
\end{aligned}
$$

from which the desired identity follows.

We are now ready to prove Theorem 1. 
Proof of Theorem 1. We will use the descending induction on $i=n$, $n-1, \ldots, 1$. In the case $i=n$ (induction base), the identity of the theorem is already shown in Lemma 5. Therefore, we assume that $i<n$ and that identity (8) is proved with $i$ replaced by $i+1$, that is,

$$
\zeta^{\star}(\underbrace{2, \ldots, 2}_{i+1}, 1, \underbrace{2, \ldots, 2}_{n-i-1}, 1)=4 \zeta^{\star}(2 i+3,2 n-2 i-1)-2 \zeta(2 n+2) .
$$

We substitute expressions

$$
\begin{aligned}
& \sum_{a_{0}>a_{i+1} \geq \cdots \geq a_{n} \geq 1} \frac{H\left(a_{0}, a_{n}\right)}{a_{0}^{2 i+1} a_{i+1}^{2} \cdots a_{n}^{2}} \\
= & \sum_{a_{0}>a_{i+1} \geq \cdots \geq a_{n} \geq 1} \frac{H\left(a_{0}+1, a_{n}\right)}{a_{0}^{2 i+1} a_{i+1}^{2} \cdots a_{n}^{2}} \\
& +\sum_{a_{0}>a_{i+1} \geq \cdots \geq a_{n} \geq 1}\left(\frac{1}{a_{0}-a_{n}}-\frac{1}{a_{0}}\right) \frac{1}{a_{0}^{2 i+1} a_{i+1}^{2} \cdots a_{n}^{2}} \\
= & \sum_{a_{0}>a_{i+1} \geq \cdots \geq a_{n} \geq 1} \frac{H\left(a_{0}+1, a_{n}\right)}{a_{0}^{2 i+1} a_{i+1}^{2} \cdots a_{n}^{2}}+2 \zeta(2 i+3,2 n-2 i-1)
\end{aligned}
$$

followed from Lemma 3 and

$$
\begin{aligned}
& \sum_{a_{0} \geq \cdots \geq a_{n} \geq 1}\left(\frac{H\left(a_{0}, a_{n}\right)}{a_{0}^{2} \cdots a_{i-1}^{2} a_{i} a_{i+1}^{2} \cdots a_{n}^{2}}-\frac{H\left(a_{0}, a_{n}\right)}{a_{0}^{2} \cdots a_{i}^{2} a_{i+1} a_{i+2}^{2} \cdots a_{n}^{2}}\right) \\
& =4 \zeta^{\star}(2 i+3,2 n-2 i-1)-4 \zeta(2 n+2)=4 \zeta(2 i+3,2 n-2 i-1)
\end{aligned}
$$

followed from Lemma 8 and (26) into the identity of Lemma 7 to get

$$
\begin{aligned}
& \sum_{a_{0} \geq \cdots \geq a_{n} \geq 1} \frac{H\left(a_{0}, a_{i}-1\right)-H\left(a_{0}, a_{i+1}-1\right)}{a_{0}^{2} \cdots a_{n-1}^{2} a_{n}}=2 \sum_{a_{0}>a_{i+1} \geq \cdots \geq a_{n} \geq 1} \frac{H\left(a_{0}+1, a_{n}\right)}{a_{0}^{2 i+1} a_{i+1}^{2} \cdots a_{n}^{2}} \\
= & 2 \sum_{a_{0} \geq a_{i+1} \geq \cdots \geq a_{n} \geq 1} \frac{H\left(a_{0}+1, a_{n}\right)}{a_{0}^{2 i+1} a_{i+1}^{2} \cdots a_{n}^{2}}-2 \sum_{a_{0} \geq a_{i+2} \geq \cdots \geq a_{n} \geq 1} \frac{H\left(a_{0}+1, a_{n}\right)}{a_{0}^{2 i+3} a_{i+2}^{2} \cdots a_{n}^{2}} .
\end{aligned}
$$

The last identity may be written as

$$
\begin{aligned}
& \sum_{a_{0} \geq \cdots \geq a_{n} \geq 1} \frac{H\left(a_{0}, a_{i}-1\right)}{a_{0}^{2} \cdots a_{n-1}^{2} a_{n}}-2 \sum_{a_{0} \geq a_{i+1} \geq \cdots \geq a_{n} \geq 1} \frac{H\left(a_{0}+1, a_{n}\right)}{a_{0}^{2 i+1} a_{i+1}^{2} \cdots a_{n}^{2}} \\
= & \sum_{a_{0} \geq \cdots \geq a_{n} \geq 1} \frac{H\left(a_{0}, a_{i+1}-1\right)}{a_{0}^{2} \cdots a_{n-1}^{2} a_{n}}-2 \sum_{a_{0} \geq a_{i+2} \geq \cdots \geq a_{n} \geq 1} \frac{H\left(a_{0}+1, a_{n}\right)}{a_{0}^{2 i+3} a_{i+2}^{2} \cdots a_{n}^{2}},
\end{aligned}
$$


where the right-hand side equals $-2 \zeta(2 n+2)$ by Lemma 4 applied to $i+1$ instead of $i$ and (26), so does the left-hand side:

$$
\sum_{a_{0} \geq \cdots \geq a_{n} \geq 1} \frac{H\left(a_{0}, a_{i}-1\right)}{a_{0}^{2} \cdots a_{n-1}^{2} a_{n}}-2 \sum_{a_{0} \geq a_{i+1} \geq \cdots \geq a_{n} \geq 1} \frac{H\left(a_{0}+1, a_{n}\right)}{a_{0}^{2 i+1} a_{i+1}^{2} \cdots a_{n}^{2}}=-2 \zeta(2 n+2) .
$$

Finally, from (18) and (27) we obtain identity (8) for the given $i$, completing the proof of Theorem 1 .

\section{Weighted sum formula: Proof of Theorems 2 and 3}

We first prove the weighted sum formula.

Proof of Theorem 3. Recall two standard ways of computing the product of two zeta values [23]: the shuffle product (coming from the representation of MZVs by iterated integrals [18])

$$
\zeta(m) \zeta(n-m)=\sum_{l=2}^{n-1}\left(\left(\begin{array}{c}
l-1 \\
m-1
\end{array}\right)+\left(\begin{array}{c}
l-1 \\
n-m-1
\end{array}\right)\right) \zeta(l, n-l)
$$

and the harmonic (or stuffle) product (originated by the series representation [8])

$$
\zeta(m) \zeta(n-m)=\zeta(n)+\zeta(m, n-m)+\zeta(n-m, m)
$$

here $m>1$ and $n>m+1$ are arbitrary integers. ${ }^{2}$ Summing up the righthand sides in (28a) and (28b) over $m=2,3, \ldots, n-2$, we get

$$
\begin{aligned}
& \sum_{l=2}^{n-1} \sum_{m=2}^{n-2}\left(\left(\begin{array}{c}
l-1 \\
m-1
\end{array}\right)+\left(\begin{array}{c}
l-1 \\
n-m-1
\end{array}\right)\right) \zeta(l, n-l) \\
& \quad=(n-3) \zeta(n)+\sum_{m=2}^{n-2}(\zeta(m, n-m)+\zeta(n-m, m)),
\end{aligned}
$$

which reduces to

$$
2 \sum_{l=2}^{n-1} \sum_{m=2}^{\min \{l, n-2\}}\left(\begin{array}{c}
l-1 \\
m-1
\end{array}\right) \zeta(l, n-l)=(n-3) \zeta(n)+2 \sum_{m=2}^{n-2} \zeta(m, n-m) .
$$

${ }^{2} \mathrm{~A}$ general form of these relations, known as double-shuffle relations, is discussed in $[6,10]$. 
Adding $2 \zeta(n-1,1)$ to the both sides of the latter equality, we obtain

$$
2 \sum_{l=2}^{n-1} \sum_{m=2}^{l}\left(\begin{array}{c}
l-1 \\
m-1
\end{array}\right) \zeta(l, n-l)=(n-3) \zeta(n)+2 \sum_{m=2}^{n-1} \zeta(m, n-m)
$$

and using the binomial theorem for the left-hand side results in

$$
2 \sum_{l=2}^{n-1}\left(2^{l-1}-1\right) \zeta(l, n-l)=(n-3) \zeta(n)+2 \sum_{m=2}^{n-1} \zeta(m, n-m) .
$$

It remains to apply Euler's formula (4) to the both sides, to arrive at the desired formula (10).

Remark 2. After reading a draft version of this paper Kentaro Ihara and Masanobu Kaneko pointed out that Theorem 3 can also be proved by using generating functions in $[6,10]$ (for instance, specializing $X=Y=1$ equality (3.14) in [6]), and, in fact, there are more weighted versions of Euler's relation (4) but they do not have such a simple form as in (10).

Proof of Theorem 2. For $i=2, \ldots, n-1$, the sum

$$
\sum_{e_{1}+e_{2}+\cdots+e_{n-i}=i-2} \zeta\left(3+e_{1}, 1+e_{2}, 1+e_{3}, \ldots, 1+e_{n-i}\right)
$$

can be written as

$$
\sum_{e_{1}+e_{2}=i-2} \zeta\left(n-i+1+e_{1}, 1+e_{2}\right)=\sum_{l=1}^{i-1} \zeta(n-l, l)
$$

by the identity in [13]. Therefore,

$$
\begin{aligned}
2^{n-2} & \zeta(3, \underbrace{1, \ldots, 1}_{n-3})+2^{n-3} \sum_{e_{1}+e_{2}+\cdots+e_{n-3}=1} \zeta\left(3+e_{1}, 1+e_{2}, \ldots, 1+e_{n-3}\right)+\cdots \\
& +2^{n-i} \sum_{e_{1}+e_{2}+\cdots+e_{n-i}=i-2} \zeta\left(3+e_{1}, 1+e_{2}, \ldots, 1+e_{n-i}\right)+\cdots+2 \zeta(n) \\
& =\sum_{i=2}^{n-1} 2^{n-i} \sum_{l=1}^{i-1} \zeta(n-l, l)=\sum_{l=1}^{n-2} \sum_{i=2}^{n-l-1} 2^{i} \zeta(n-l, l) \\
& =\sum_{l=1}^{n-2}\left(2^{n-l}-2\right) \zeta(n-l, l) .
\end{aligned}
$$


Applying Euler's formula (4), the latter sum becomes

$$
\sum_{l=1}^{n-2} 2^{n-l} \zeta(n-l, l)-2 \zeta(n),
$$

which turns out to be $(n-1) \zeta(n)$ by Theorem 3 . Finally, we use the formula

$$
(n-1) \zeta(n)=\zeta^{\star}(2, \underbrace{1,1, \ldots, 1}_{n-2}),
$$

which is a special case of the sum formula due to Granville [7] and Zagier [19]. This implies (9) and completes our proof of Theorem 2.

As one can see from the above proofs of Theorems 2 and 3, these theorems are equivalent modulo the algebraic relations from [13] (which also contain the sum formula as a particular case).

\section{Acknowledgment}

We would like to thank Kentaro Ihara, Masanobu Kaneko, Jun-ichi Okuda and Don Zagier for useful comments. We are also thankful to Tatsushi Tanaka for providing us with a program of computing multiple zeta star values which was used in our experimental verification of the formulae. We are indebted to the two anonymous referees of the journal for their helpful remarks.

The work was done when both the authors visited in the Max Planck Institute for Mathematics (Bonn). We thank the staff of the institute for the wonderful working conditions we experienced there.

The work of Yasuo Ohno was supported by a special programme of the Kinki University (Osaka). The work of Wadim Zudilin was supported by a fellowship of the Max Planck Institute for Mathematics (Bonn).

\section{References}

[1] T. Aoki and Y. Ohno, Sum relations for multiple zeta values and connection formulas for the Gauss hypergeometric functions, Publ. Res. Inst. Math. Sci. 41 (2005), 329-337.

[2] D.J. Broadhurst and D. Kreimer, Association of multiple zeta values with positive knots via Feynman diagrams up to 9 loops, Phys. Lett. B 393 (1997), 403-412. 
[3] P. Deligne and A.B. Goncharov, Groupes fondamentaux motiviques de Tate mixte, Ann. Sci. École Norm. Sup. (4) 38 (2005), 1-56.

[4] V.G. Drinfel'd, On quasitriangular quasi-Hopf algebras and on a group that is closely connected with $\operatorname{Gal}(\overline{\mathbb{Q}} / \mathbb{Q})$, Algebra i Analiz (1990), 149-181; Leningrad Math. J. 2 (1991), 829-860 (English transl.).

[5] L. Euler, Meditationes circa singulare serierum genus, Novi Comm. Acad. Sci. Petropol 20 (1775), 140-186; Opera Omnia, Ser. I, vol. 15, Teubner, Berlin, 1927, 217-267 (Reprinted).

[6] H. Gangl, M. Kaneko and D. Zagier, Double zeta values and modular forms, Automorphic forms and zeta functions, Proceedings of the Conference in Memory of Tsuneo Arakawa, World Sci. Publ., Hackensack, NJ, 2006, 71-106.

[7] A. Granville, A decomposition of Riemann's zeta-function, Analytic number theory (Kyoto, 1996), London Mathematical Society Lecture Note Series, 247, Cambridge University Press, Cambridge, 1997, 95-101.

[8] M.E. Hoffman, Multiple harmonic series, Pacific J. Math. 152 (1992), 275-290.

[9] M.E. Hoffman and Y. Ohno, Relations of multiple zeta values and their algebraic expression, J. Algebra 262 (2003), 332-347.

[10] K. Ihara, M. Kaneko and D. Zagier, Derivation and double shuffle relations for multiple zeta values, Compos. Math. 142 (2006), 307-338.

[11] C. Kassel, Quantum groups, Graduate Texts in Mathematics, 155, Springer-Verlag, New York, 1995.

[12] D. Kreimer, Knots and Feynman diagrams, Cambridge Lecture Notes in Physics 13, Cambridge University Press, Cambridge, 2000.

[13] Y. Ohno, A generalization of the duality and sum formulas on the multiple zeta values, J. Number Theory 74 (1999), 39-43.

[14] Y. Ohno and J. Okuda, On the sum formula for the q-analogue of nonstrict multiple zeta values, Proc. Amer. Math. Soc. 135 (2007), 30293037.

[15] Y. Ohno and N. Wakabayashi, Cyclic sum of multiple zeta values, Acta Arith. 123 (2006), 289-295. 
[16] T. Terasoma, Mixed Tate motives and multiple zeta values, Invent. Math. 149 (2002), 339-369.

[17] E.A. Ulanskii, Identities for generalized polylogarithms, Mat. Zametki 73 (2003), 613-624; Math. Notes 73 (2003), 571-581 (English transl.).

[18] D. Zagier, Values of zeta functions and their applications, First European Congress of Mathematics, vol. II (Paris, 1992), Progress in Mathematics, 120, Birkhäuser, Basel, 1994, 497-512.

[19] D. Zagier, Multiple zeta values, Unpublished manuscript, Bonn, 1995.

[20] S.A. Zlobin, On some integral identities, Uspekhi Mat. Nauk (2002), 153-154; Russian Math. Surveys 57 (2002), 617-618 (English transl.).

[21] S.A. Zlobin, Expansion of multiple integrals in linear forms, Mat. Zametki 77 (2005), 683-706; Math. Notes 77 (2005), 630-652 (English transl.).

[22] S.A. Zlobin, Generating functions for the values of a multiple zeta function, Vestnik Moskov. Univ. Ser. I Mat. Mekh. no. 2 (2005), 55-59; Moscow Univ. Math. Bull. 60 (2005), 44-48 (English transl.).

[23] W. Zudilin, Algebraic relations for multiple zeta values, Uspekhi Mat. Nauk (2003), 3-32; Russian Math. Surveys 58 (2003), 1-29 (English transl.).

Department of Mathematics

Kinki University

Higashi-OSAKA

OSAKA 577-8502

JAPAN

E-mail address: ohno@math.kindai.ac.jp

Department of Mechanics and Mathematics

Moscow Lomonosov State University

Vorobiovy Gory, GSP-1

119991 Moscow

RUSSIA

Steklov Mathematical Institute

Russian ACADEMy of ScIEnces

GUBKINA STR. 8

119991 Moscow

Russia

E-mail address: wadim@mi.ras.ru

Received November 11, 2007 
